\title{
Maximum Allowable Transfer Interval Aware Scheduling for Wireless Remote Monitoring
}

\author{
Mounssif Krouka, Anis Elgabli, and Mehdi Bennis \\ Centre for Wireless Communications \\ University of Oulu, Oulu, Finland
}

\begin{abstract}
In this paper, we tackle the problem of remote monitoring (e.g., remote factory) in which a number of sensor nodes are transmitting time sensitive measurements to a remote monitoring site. We assume that packets generated by different sensors have different sizes. Moreover, different sensors have different Maximum Allowable Transfer Intervals (MATIs). We consider minimizing a metric that maintains a trade-off between minimizing the average MATI violation of all sensors, and minimizing the probability that the MATI violation of each sensor exceeds a predefined threshold. We formulate the problem as a stochastic optimization problem with integer constraints. In order to solve this problem, we first relax the original intractable formulation to a tractable problem. Then, we use the Lyapunov stochastic optimization framework to solve the relaxed problem. Simulation results show that the proposed algorithm outperforms the considered baselines in terms of minimizing the probability of the MATI violation for all sensors.

Index Terms-Wireless Networked Control Systems, URLLC,
\end{abstract} Stochastic Optimization, loT, 5G

\section{INTRODUCTION}

Recent developments in wireless networks and the evolution of $5 \mathrm{G}$ applications such as the Internet of Things (IoT) enabled a massive number of devices to be connected. This allows a real time human interaction with the physical world under various applications such as remote surgery, tactile internet and industrial automation [1]. Industrial Internet of Things (IloT) allows remote devices to sense, actuate and control physical plants. First, sensors transmit measured data from physical plants to a central monitoring/controlling system over a wireless network, then based on the received information, the controller sends control commands to the remote actuators [2].

However, in control systems, sensing information and control commands must be delivered under Ultra Reliability and Low Latency (URLLC) constraints, which is a challenging problem when communication is over wireless links [3]. Moreover, in industrial automation, the typical playload size ranges from 40 to 250 bytes [4]. Thus, using Shannon's channel capacity for system analysis, which assumes infinite transmission blocklength, is not appropriate. To tackle this issue, the finite blocklength regime's transmission rate [5] was addressed in [6], [7]. One metric that has been widely used to relate the control system's stability to the rate by which messages are exchanged between the control and the plant is the Maximum Allowable Transfer Interval (MATI) [8]. MATI is the upper bound on the interval between two successful packet receptions that guarantees system stability. In other words, to ensure the system stability and avoid running into

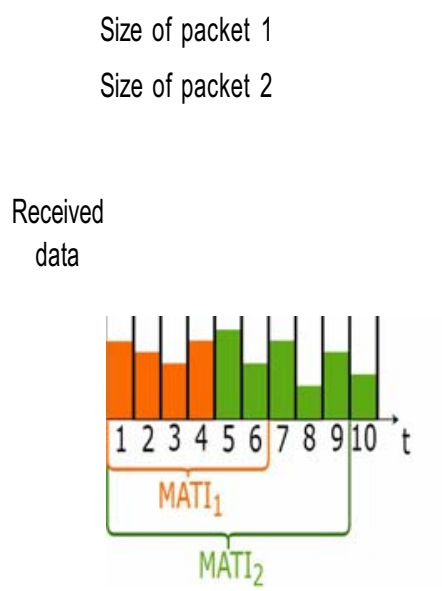

(a) Packet level scheduling
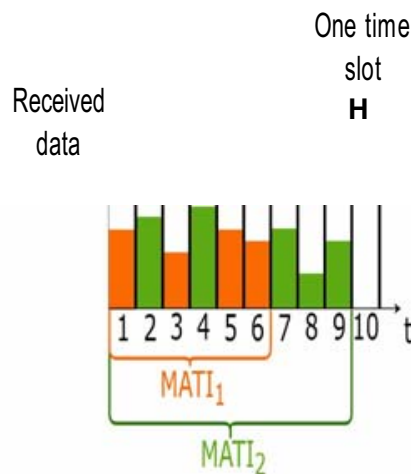

(b) Time slot scheduling

Fig. 1: Illustrative example comparing packet level and time slot level scheduling.

unstable control system scenarios, at least one packet should be received within the MATI. Recently, many works have tackled the problem of scheduling sensors and optimizing resources for wireless remote monitoring/controlling while ensuring information freshness. One commonly used metric is the Age of Information (Aol) which measures the elapsed time since the last information update was received at the controller [9]. In [10], a joint time slot scheduling and sampling policy was proposed for an loT monitoring system with non-uniform status updates while minimizing Aol. [11] optimized both wireless energy transfer and scheduling updates from IoT devices while minimizing the long-term weighted sum Aol at the destination node. However, URLLC scenarios require moving beyond average-based metrics. i,e. the works in [10], [11] only considered minimizing the average Aol which is ill-suited to guarantee high reliability. This is because a few number of successive outdated packets can cause tremendous performance degradation especially in automated factories.

On the other hand, the authors of [12] proposed a Reinforcement Learning algorithm for scheduling sensors with different packet sizes in an industrial automation scenario. Furthermore, they considered URLLC requirements by minimizing the sum Aol of all sensors while keeping the probability that the age of every sensor exceeds a predefined threshold low. However, since this work considered scheduling at the packet 
level, scheduling long packets can prevent sensors with short packets to meet their transmission deadlines, which affects the reliability of the system.

MATI was studied in [13] where the authors formulated a joint optimization problem of power control, rate and scheduling, in which different sensors transmit packets with different sizes. Moreover, the work of [14] proposed a general protocol for both sensor to controller and controller to actuator connections over a multihop network that satisfies the MATI requirements and schedulability of resources. Nevertheless, incorporating MATI with URLLC was not studied in these works.

The main contribution of our work is to minimize a performance metric that is composed of two terms: (i) the average of the MATI violation of all sensors, and (ii) the probability of the MATI violation of each sensor. In particular, we schedule multiple sensors at the time slot level. This enables sensors with different packet sizes to maintain their transmission time within the MATI constraint. Fig. 1 illustrates the advantage of the time slot scheduling over the packet level scheduling. We consider sensor 1 with short packet size and tight MATI and sensor 2 with long packet size and large MATI. In the case of packet level, the controller keeps scheduling one sensor until its full packet is received. We notice that when sensor 1 is scheduled, sensor 2 needs to wait for the reception of packet 1 and that prevents packet 2 from meeting its deadline. This incurs MATI violations that degrade the overall system reliability. On the other hand, time slot scheduling allocates the bandwidth at every time slot. Thereby, instead of waiting for packet 1 to be delivered, the controller alternatively schedules the sensors every time slot such that sensor 2 avoids running into the MATI violation. We formulate our problem as a stochastic discrete (non-convex) optimization problem. In order to solve it, we relax the non-convexity constraint, and apply the Lyapunov stochastic optimization framework with successive convex approximation to approximate the solution of the original proposed optimization problem.

The rest of the paper is organized as follows. In section II we describe the system model. In section III we present our problem formulation and define our proposed framework. In section IV, we present the simulation results and discussion. Finally, in section $\mathrm{V}$ we conclude the paper.

$$
\text { II. System Model }
$$

We consider a factory monitoring scenario consisting a set $\mathrm{N}$ of $\mathrm{N}$ sensors that collect and transmit real-time data packets to a monitoring/controlling unit over wireless links. The controller schedules $\mathrm{M}<\mathrm{N}$ sensors for transmission by allocating orthogonal channels. Moreover, we assume that sensors generate data packets at different sizes where sensor $\mathrm{n} \in\{1, \cdots, N\}$ generates packets of size $D \mathrm{n}$. We suppose that the timeline is divided into equal and discrete time slots indexed by $t=1,2, \cdots$. Thus, one full packet transmission may take several time slot durations. We assume a block fading wireless channel that is invariant for every time slot duration. We also assume that the controller has a perfect Channel
State Information (CSI) at each time slot from all sensors. Let $R h(L n, e)$ denotes the achievable finite blocklength rate of sensor $\mathrm{n}$ at time slot $\mathrm{t}$, where $\mathrm{Ln}$ and eare the blocklength for sensor $\mathrm{n}$ and the decoding error, respectively. Thus, $R f(\mathrm{Ln}, \mathrm{e})$ is defined as follows [5]:

$$
\mathrm{R},(\operatorname{Ln}, \mathrm{e})=\mathrm{Bn}(<)[\log , \mathrm{a}+\mathrm{N} \text { i l })-/ \mid<\mathrm{r} 1(\mathrm{e})
$$

where $B n(t)$ is the assigned bandwidth to sensor $n$ at time slot $t, P n$ is the transmission power of sensor $n, h n(t)$ is the channel gain including the distance dependent path loss and the channel fading between the controller and sensor $\mathrm{n}$ at time slot t. N0 is the power spectral density of the additive white Gaussian noise. $V=77 \mid) 2++Y 7 \mid 0+I(\operatorname{loge}) 2$ is the channel dispersion, where $\left.r n(t)=N^{\wedge} r T e t\right)$ is the Signal to Noise Ratio (SNR), and Q-1 () is the inverse Q-function [5]. To simplify the notation, we will refer to the finite blocklength rate of sensor $n$ at time slot $t$ as $R n(t)$ throughout the rest of the paper.

Let $r n(t)$ be the cumulative data size in bytes received at the controller from the beginning until time slot $t$ from sensor $n$. $r n(t)$ evolves according to the following equation.

$$
r n(t)=r n(t-1)+r R n(t)
$$

where ${ }_{T}$ is a one time slot duration.

We denote by $T n$ the MATI duration for sensor $n$. let $S n(t)$ be the number of bytes received at the controller from sensor $\mathrm{n}$ in the time between $\mathrm{t}$ and $(\mathrm{t}-\mathrm{Tn})$. Mathematically $\mathrm{Sn}(\mathrm{t})$ is defined as follows:

$$
S_{n}(t)=r_{n}(t)-r_{n}\left(t-T_{n}\right),
$$

Let $\ln (\mathrm{t})$ be the MATI violation of sensor $n$ at time slot $t$. Note that $\operatorname{In}(\mathrm{t})$ depends on $\mathrm{Sn}(\mathrm{t})$ and this relation can be mathematically captured by the following equation:

$$
\begin{array}{ll} 
& 0 \\
\ln (t) & \text { if } S_{n}(t)>D_{n} \\
1 & \text { if } S_{n}(t)<D_{n}
\end{array}
$$

Equation (4) states that for the event $I_{n}(t)=0$ to occur, we need to insure $S_{n}(t)>D_{n}$ for every $t$.

III. MATI-AWARE SENSOR SCHEDULING PROBLEM

It is important to maintain low MATI violations over the sensors for stability. Therefore, we focus on minimizing the average MATI violations while maintaining the probability that the MATI violation of each sensor exceeding zero is kept below a predefined threshold $a$. This probability can be expressed as: $p r \ln (t)=1<a \quad V n$. Note that the occurrence of the event $I_{n}(t)>0$ is associated with the event $S_{n}(t)<D_{n}$. Thus, the MATI violation probability can be replaced by $\operatorname{Pr}\left(\mathrm{S}_{\mathrm{n}}(\mathrm{t})<\mathrm{D}_{\mathrm{n}}\right)<\mathrm{a} \quad \mathrm{Vn}$. In order to account 
for such objective and constraint, we consider the following optimization problem.

$$
\begin{aligned}
& \mathrm{N} \mathrm{T} \\
& \underset{B n(t)}{\operatorname{minimize}} \quad \mathrm{T}_{\text {。 } 。} \quad \mathrm{~T}_{\mathrm{n}=1 \mathrm{t}=1} \ln (\mathrm{t}) \\
& \text { subject to }(1(-(4) \\
& \operatorname{Pr}(\mathrm{Sn}(\mathrm{t})<\mathrm{Dn})<a \quad \mathrm{Vn} \\
& \mathrm{N} \\
& \mathrm{V} \quad \mathrm{Bn}(\mathrm{t})<\mathrm{Bmax}, \quad \mathrm{Vt} \\
& \mathrm{Bn}(\mathrm{t})>0, \quad \quad \mathrm{Vn}, \mathrm{Vt}
\end{aligned}
$$

where B max is the total available bandwidth.

\section{A. Lyapunov Optimization-Based Scheduling Algorithm}

The problem defined in (5) is a stochastic optimization problem with non-convex constraints (4), (5c) which is hard to solve. Therefore, we first relax the formulation to a simpler problem in order to find a tractable solution using stochastic optimization methods, and then use the Lyapunov framework to solve it. From (4), we know that $I_{n}(t)$ is a binary (nonconvex) variable. Hence, the first step is to minimize the convex relaxation of $\mathrm{In}_{\mathrm{n}}(\mathrm{t})$. Therefore, we minimize $/ \mathrm{n}(\mathrm{t})$ which is defined as follows:

$$
\operatorname{In}(t)=\left[D_{n}-S_{n}(t)\right]+,
$$

where $[x]+=\max (x, 0)$. Fig. 2 plots the dependence of $I_{n}(t)$ as a function of $S_{n}(t)$. It can be noted that $I_{n}(t)$ decreases as $S_{n}(t)$ increases, until $S_{n}(t)$ reaches $D_{n}$, where it remains zero from that point onwards. Here, $S_{n}(t)>D_{n}$ does not contribute in decreasing $I_{n}(t)$ meaning that scheduling resources to push more sensors to achieve $S_{n}(t)>D_{n}$ is more preferable than increasing $S_{n}(t)$ for the sensors that already have $S_{n}(t)>D_{n}$. Hence, the problem in (5) can be well approximated by the following formulation:

$$
\begin{array}{ll}
\underset{B n(t)}{\operatorname{maximize}} & A{ }^{N} T_{n=1 t=1} S n(t) \\
\text { subject to } & (1)-(3),(5 c)-(5 e) .
\end{array}
$$

Since $S n(t) \quad r n(t) \quad r n(t \quad T n)$, and $R n(t \quad T n)$ are already given at time slot $t$, (7a) is equivalent to maximizing

$\mathrm{N}=1 \mathrm{~T}=1 \mathrm{rn}(\mathrm{t})$, which is in turn equivalent to maximizing $J 2 n=\wedge 2$ 1=1Rn(t). Therefore, our equivalent problem formulation becomes:

$$
\begin{array}{cc}
\underset{B n(t)}{\operatorname{maximize}} & T^{N} \propto T_{n=1 t=1} R n(t) \\
\text { subject to } & (1)-(3),(5 c)-(5 e) .
\end{array}
$$

\section{B. Proposed Solution Based on Lyapunov Framework}

To solve the stochastic optimization problem in (8) we use the tools form Lyapunov optimization framework, as presented next. First, all time average constraints must be converted into virtual queues. Henceforth, the reliability constraint in $(5 c)$

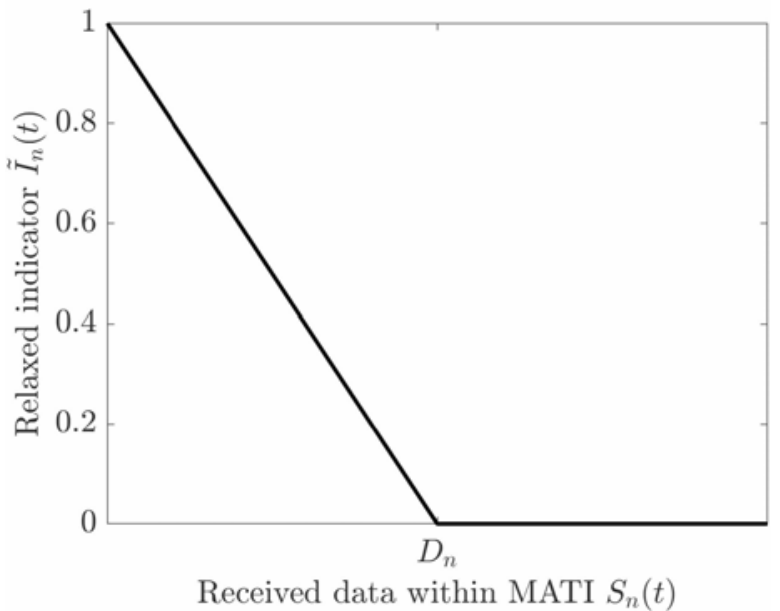

Fig. 2: Evolution of $\mathrm{In}(\mathrm{t})$ with respect to received data $\mathrm{Sn}(\mathrm{t})$.

can be expressed as $E[1(s n<D n)]<$ a for each sensor $n$, and a virtual queue $A_{n}$ is introduced with the following dynamics [15].

$$
A n(t+1) \quad A n(t)+\left(I\left(S_{n}(t)<D_{n}\right)-a\right)^{1+}
$$

Let $A(t)=[A n(t)] n G N$, we define the Lyapunov function in quadratic form as $L(A(t))=, A(t) T A(t)$. The one-slot drift of the Lyapunov function is $A L t=L(A(t+1))-L(A(t))$.

$$
\left.A L t=\begin{array}{ll}
1 & N \\
2 & X]
\end{array}\right][A n(t+1)-\quad(t)] .
$$

Using the relation,

$$
(t+1)<(t)+\left(I\left(S_{n}(t)<D_{n}\right)-e\right) 2+2 A n(t)\left(1\left(\operatorname{Sn}(t)<D_{n}\right)-e\right) .
$$

The term $\left(1\left(S_{n}(t)<D_{n}\right)-e\right) 2$ is bounded when queue stability is satisfied. Hence, this term is replaced by a bounded value $U$ as follows:

$$
A L t<U+\bigwedge_{n=1}^{N} A n(t)\left(I\left(S_{n}(t)<D_{n}\right)-e\right) .
$$

We define the conditional expected Lyapunov drift at time slot $\mathrm{t}$ as $\mathrm{E}\left[\mathrm{L}(\mathrm{A}(\mathrm{t}+1))-\mathrm{L}\left(\mathrm{A}\left({ }^{*}\right)\right) \mid \mathrm{A}(\mathrm{t})\right]$. Let $W>0$ be a scalar variable that controls the tradoff between the accuracy of the solution of (8) and the queue length. By bringing the penalty term $W E[R n(t) \mid A(t)]$ to the inequality (12), we minimize the upper bound of the drift plus penalty $E[A L t \mid A(t)]-W E[R n(t) \mid A(t)]$. Consequently, our goal is to solve the following:

$$
\begin{aligned}
& \text { N } \\
& \text { minimize }-W R n(f)+V A n(f)\left(I\left(S_{n}(t)<D_{n}\right)\right) \\
& B_{n}(t) \\
& \text { subject to (1)-(3), (5d)-(5e), (9). }
\end{aligned}
$$

In our simulation setting, we assume that the SNR » 1 . This reduces the second term of the rate expression in (1) to $-B n(t)^{\wedge}\left({ }^{\top} g_{r} e\right) 2 Q-1(e)$. Moreover, $1\left(S_{n}(t)<D\right.$, ) is a binary (non-convex) variable, we introduce a new variable $\mathrm{Zn}(\mathrm{t})$ 


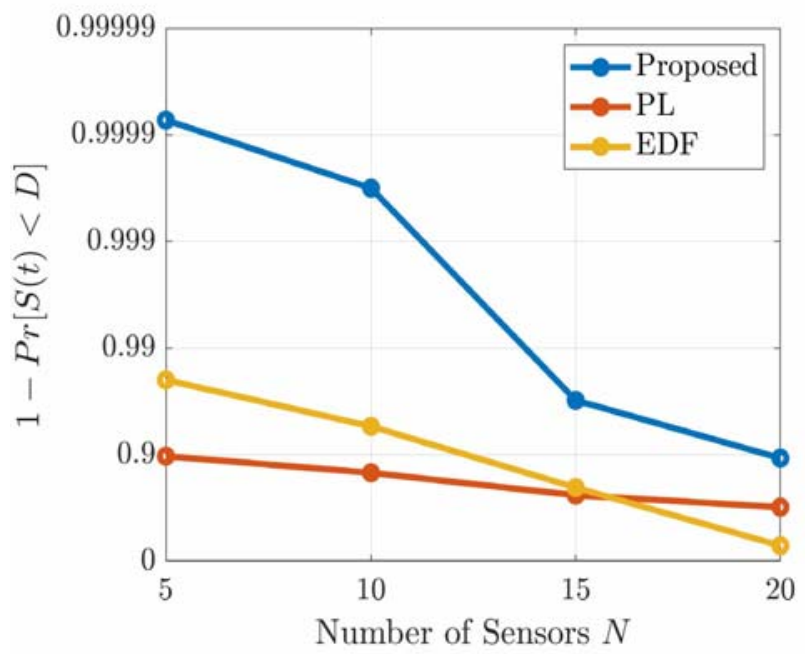

Fig. 3: CDF of the received data within the MATI for (a) sensor 1 , (b) sensor 5 , and (c) sensor 10.

such that $Z n(t)>1(S n(t)<D n)$. Hence, our problem can be rewritten as follows:

$$
\begin{array}{cl}
\underset{B_{n}(t)}{\operatorname{minimize}} & -\operatorname{WRn}(t)+{ }_{n=1}^{N} A n(t) Z n(t) \\
\text { subject to } & (1)-(3),(5 d)-(5 e),(9), \\
& Z n(t)>1\left(S_{n}(t)<D_{n}\right) \quad \text { Vn- }
\end{array}
$$

Now, we replace $1(\mathrm{Sn}(\mathrm{t})<\mathrm{Dn})$ by a continuous approximation $f(S n(t))=1+e a(S 1(t)-D n)$. Since this approximation is nonconvex, we use successive convex approximation methods to solve (14). Therein, at each iteration $i, f(S n(t))$ is replaced by its first order approximation over initial choice Sn,

$$
1+\mathrm{e} \ll\left(S_{n}(t) \cdot D_{n}\right) \quad A o(S n)+A_{1}(S n) S n(t),
$$

where $A o(S n)=f(S n)$ and $A_{1}(S n)=f^{\prime}(S n)$, respectively. This approximation converts (14c) into a linear constraint, in which (14) becomes a convex optimization problem. The solution $S * 1$ is used as the initial choice $S 0+1$ until convergence, i.e., $\left|S^{*} m-S^{*}\right|<$ e.

\section{Numerical Results}

To validate our proposed scheduling algorithm numerically, we use a set of simulations where we consider a group of 5 sensor clusters with every cluster containing 1 to 4 sensors. i.e, the total number of sensors varies from 5 to 20 . The sensors of each cluster generate packets of sizes 50 to 250 bytes, with a 50 bytes step size. The clusters have different MATI requirements. i.e, we vary Tn from 8 to $40 \mathrm{~ms}$ with a step size of $8 \mathrm{~ms}$. The communication environment is simulated for a factory (indoor) scenario with the path loss model: $33 \log r+20 \log f+32(\mathrm{~dB}), r$ is the distance from the controller, and $f$ is the carrier frequency. In our simulation $f=2.625 \mathrm{GHz}$ [16]. The other simulation parameters are: $\mathrm{N} 0=-174 \mathrm{dBm} / \mathrm{Hz}, B \max =1 \mathrm{MHz}, \mathrm{W}=1, \mathrm{\tau}=1 \mathrm{~ms}$, $\mathrm{Ln}=500, \mathrm{e}=10-5, \mathrm{P}=1 \mathrm{~mW}, \mathrm{a}=10-3, \mathrm{a}=1$, $e=10-3$, and $r=5 \mathrm{~m}$. According to these choices, sensors of cluster 1 have the tightest MATI and shortest packet size. On the other hand, sensors of cluster 5 have the largest MATI and the longest packet size. We run simulations over a large number of realizations. Our proposed scheduling algorithm allocates orthogonal channels for $\mathrm{M}<\mathrm{N}$ sensors at every time slot. This enables allocating more bandwidth for sensors with different packet sizes and MATIs when necessary to guarantee a minimum MATI violation.

On the other hand, we consider two baselines for comparison. The first baseline considers scheduling sensors at the packet level. i.e, the controller schedules multiple sensors and maintains the same allocated bandwidth for every sensor until packets from all sensors have been received. We refer to this baseline as packet level "PL". Baseline two considers scheduling one sensor at a time until its packet is successfully received at the controller side. It follows the "Earliest Deadline First" strategy by scheduling the closest sensor to violate its MATI. We refer to this baseline as "EDF".

Fig. 3 plots the reliability, which is the probability of receiving a packet within the MATI, as a function of the total number of sensors. For every choice of the number of sensors, we plot the worst reliability obtained by a sensor. It is noted that our proposed algorithm provides more reliable communication compared to the baselines. For $\mathrm{N}=5$, our algorithm reaches $99.991 \%$ reliability compared to $96.32 \%$ achieved by EDF and $88.86 \%$ by PL. In the case of $\mathrm{N}=10$, the proposed algorithm reaches the reliability of $99.93 \%$ while it is $92.40 \%$ and $74.83 \%$ for EDF and PL, respectively. We notice that as the number of sensors increases, the reliability decreases. The reason is that, the more sensors need to be scheduled, the less bandwidth will be allocated per sensor. This decreases the rate, which in turn increases the transmission time that leads to more MATI violations. We observe that our algorithm is more robust at large number of sensors. From $\mathrm{N}=15$ onwards, we see that the reliability of EDF drops drastically below the PL. This is due to the fact that EDF focuses on delivering packets for sensors with tight MATIs, which incurs very high MATI violations for sensors with larger MATIs. One way to maintain system reliability above $99.9 \%$ is to increase the transmit power.

Fig. 4a-c plots the CDF of Sn with $N=10$ for sensors 1,5 and 10 , respectively. The results show the performance improvement of our proposed solution over both baselines for different packet size cases. As can be seen in Fig. 4a, our algorithm shows that $99.94 \%$ of packets were successfully received within their MATIs. Whereas a reliability of $98.33 \%$, and $74.83 \%$ is achieved for EDF and PL, respectively. In the cases of Fig. 4b and Fig. 4c, our algorithm reaches a reliability of $99.94 \%$ and $99.93 \%$, respectively. Results from both Fig. $4 \mathrm{~b}$ and Fig. 4c illustrate the stability of our algorithm for medium and long packet scenarios. Note that PL performs worse than EDF. This is due to the fact that for PL, the controller updates the bandwidth allocation decision only after receiving packets from all sensors. Therefore, while waiting for the last packet to be delivered, multiple short packets may have encountered 

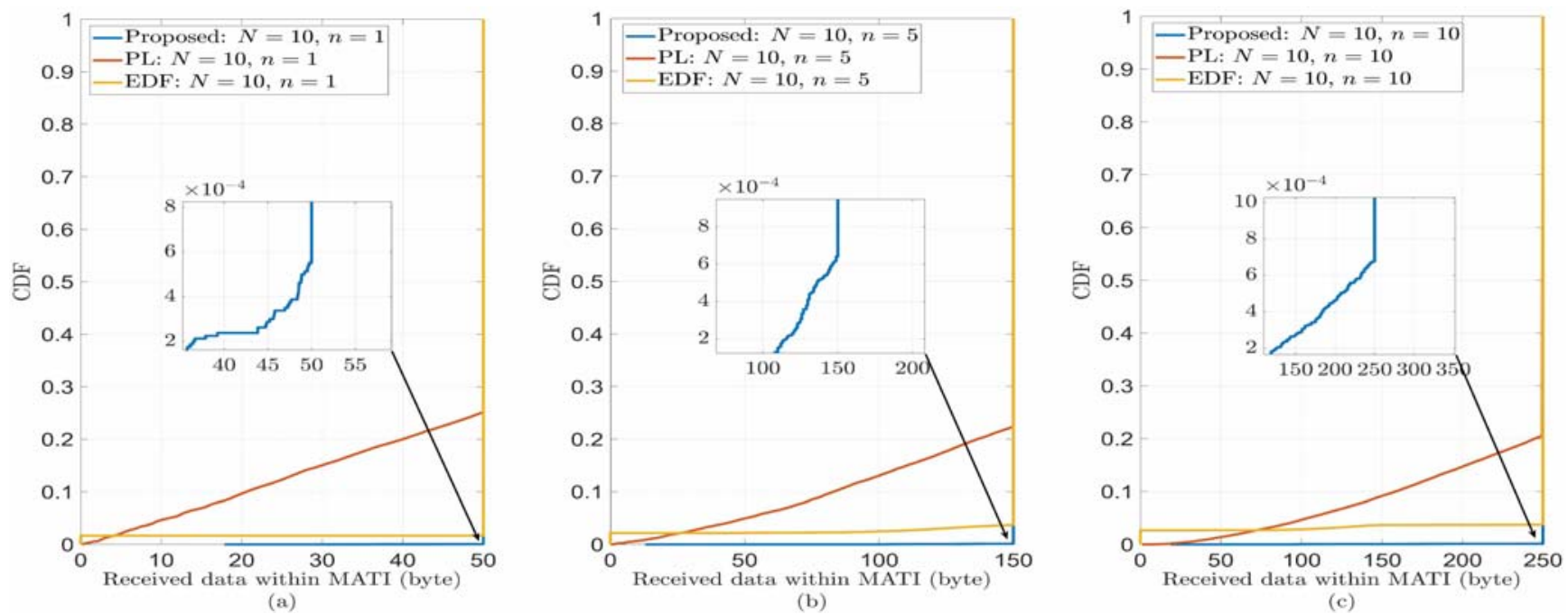

Fig. 4: CDF of the received data within the MATI for (a) sensor 1, (b) sensor 5, and (c) sensor 10.

MATI violations. From the CDF plots, we see that there are events when no data is received within the MATI for the baseline EDF. This is because EDF algorithm selects one sensor for transmission at a time. Thus, some sensors may violate their MATIs while waiting to be scheduled.

Fig. 5a-c plots the probability that packet transmission time is greater than time $t$ for one sensor from cluster 1, 3 and 5 , respectively. The results compare the performance of our algorithm with both baselines for $N E\{5,15\}$. A is the time needed to fully transmit one packet to the controller. In Fig. $5 a$, for $\mathrm{N}=5$, EDF has a probability of $0.002 \%$ that packet transmission time exceeds the MATI, whereas $0.005 \%$ is obtained by our algorithm, and $12.95 \%$ by PL. We see that the high performance of EDF for the sensor with shortest packet and tightest MATI comes at the cost of performance degradation for the other sensors with longer packet sizes and larger MATIs, as depicted in Fig. 5b and Fig. 5c. The reason is that EDF assigns the whole bandwidth when scheduling one sensor at a time. This increases the transmission rate, which in turn decreases the packet transmission time. Moreover, the sensor with tight MATI reaches its deadline faster and this makes the EDF algorithm tend to schedule it more frequently than other sensors with larger MATIs. This is observed in Fig. $5 b$ where the performance is compared given MATI $=24 \mathrm{~ms}$. Our algorithm maintains a probability of $0.004 \%$ for packets with transmission time larger than the MATI. For the baselines, EDF obtained $2.82 \%$, while for $P L$ it is $9.31 \%$, for $N=5$. The performance limitation of EDF reflects the fact that the sensor is less frequently scheduled compared to other sensors with tighter MATIs. This results in a long delay for the sensor to be scheduled which increases the packet transmission time. In Fig. 5c, the results for the last sensor (the one having the longest packet size and largest MATI) are plotted. We can see that our algorithm outperforms the baselines, with probability of $0.008 \%$ and $0.48 \%$ of exceeding the MATI for both $\mathrm{N}=5$ and 15 , respectively.
V.

CONCLUSION AND FUTURE WORK

In this paper, we proposed an algorithm to schedule and allocate resources in a factory scenario by minimizing the MATI violation from the sensor to the controller. Our algorithm allocates resources for sensors at every time slot in order to meet URLLC constraints for all sensors. Results show that our algorithm outperforms the baselines in terms of system reliability when sensors with different packet sizes and MATIs are scheduled. For future work, we will consider the joint optimization of both bandwidth and power for packets with stochastic arrivals.

\section{References}

[1] G. Zhao, M. A. Imran, Z. Pang, Z. Chen, and L. Li, "Toward real-time control in future wireless networks: Communication-control co-design," IEEE Communications Magazine, vol. 57, no. 2, pp. 138-144, February 2019.

[2] B. Park, J. Nah, J.-Y. Choi, I.-J. Yoon, and P. Park, "Robust wireless sensor and actuator networks for networked control systems," Sensors, vol. 19, no. 7, p. 1535, Mar 2019.

[3] M. Bennis, M. Debbah, and H. V. Poor, "Ultrareliable and low-latency wireless communication: Tail, risk, and scale," Proceedings of the IEEE, vol. 106, no. 10, pp. 1834-1853, Oct 2018.

[4] " $5 \mathrm{~g}$ alliance for connected industries and automation, " $5 \mathrm{~g}$ for connected industries and automation," 5g-acia," March 2019.

[5] Y. Polyanskiy, H. V. Poor, and S. Verdu, "Channel coding rate in the finite blocklength regime," IEEE Transactions on Information Theory, vol. 56, no. 5, pp. 2307-2359, May 2010.

[6] Y. Hu, M. Serror, K. Wehrle, and J. Gross, "Finite blocklength performance of cooperative multi-terminal wireless industrial networks," IEEE Transactions on Vehicular Technology, vol. 67, no. 7, pp. 5778-5792, July 2018.

[7] B. Chang, "Qos-constrained area spectral efficiency in ultra-reliable and low-latency industrial wireless networks," pp. 1-4, Dec 2017.

[8] P. Park, S. Coleri Ergen, C. Fischione, C. Lu, and K. H. Johansson, "Wireless network design for control systems: A survey," IEEE Communications Surveys Tutorials, vol. 20, no. 2, pp. 978-1013, Secondquarter 2018.

[9] M. K. Abdel-Aziz, C.-F. Liu, S. Samarakoon, M. Bennis, and W. Saad "Ultra-reliable low-latency vehicular networks: Taming the age of information tail," 2018 IEEE Global Communications Conference (GLOBECOM), Dec 2018.

[10] B. Zhou and W. Saad, "Minimum age of information in the internet of things with non-uniform status packet sizes," 2019. 


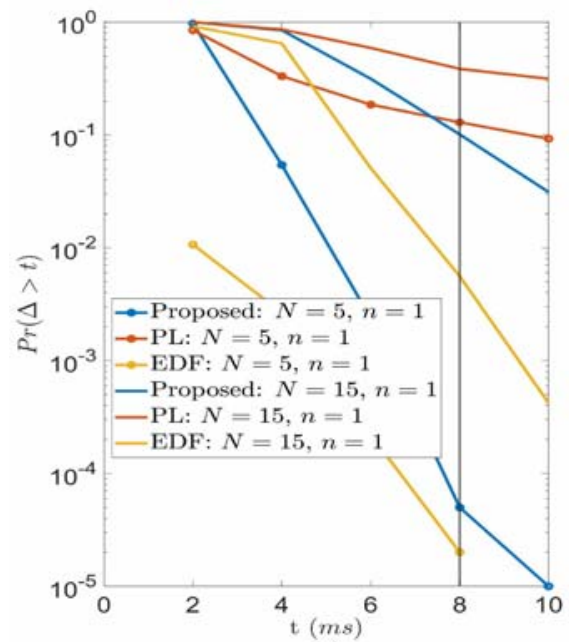

(a)

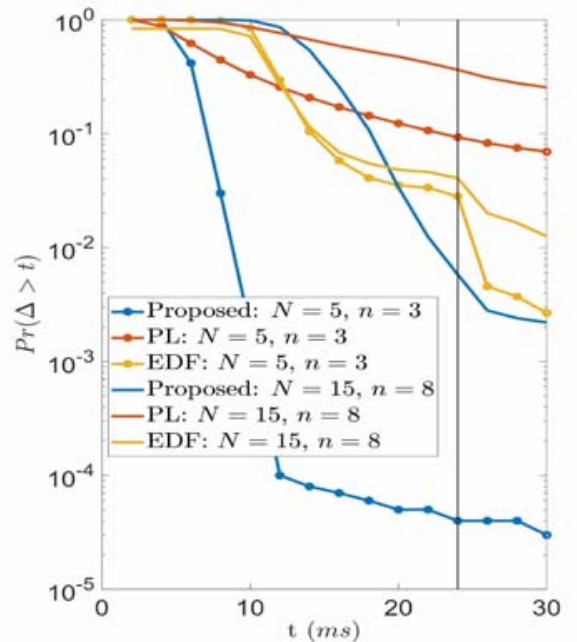

(b)

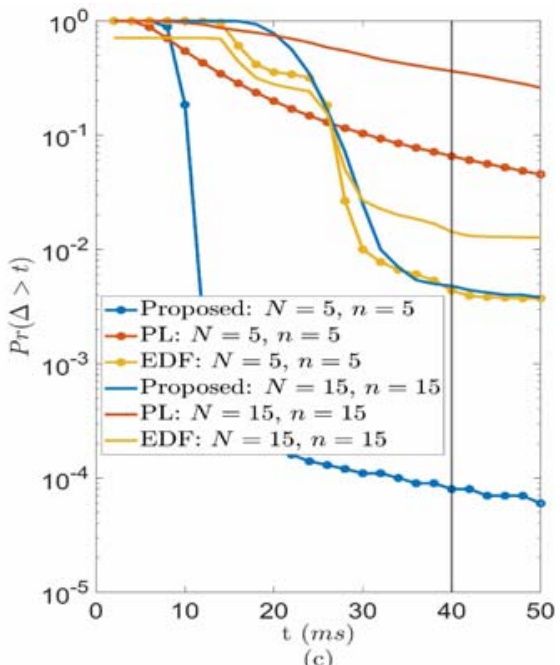

(c)

Fig. 5: Probability that the packet transmission time is greater than time $t$ with (a) MATI $=8 m s$, (b) MATI $=24 m s$, and (c) MATI $=40 \mathrm{~ms}$.

[11] M. A. Abd-Elmagid, N. Pappas, and H. S. Dhillon, "On the role of age-of-information in internet of things," 2018.

[12] A. Elgabli, H. Khan, M. Krouka, and M. Bennis, "Reinforcement learning based scheduling algorithm for optimizing age of information in ultra reliable low latency networks," 2018.

[13] Y. Sadi and S. Coleri Ergen, "Energy and delay constrained maximum adaptive schedule for wireless networked control systems," IEEE Transactions on Wireless Communications, vol. 14, no. 7, pp. 3738-3751, July 2015

[14] P. Park, P. D. Marco, and K. H. Johansson, "Cross-layer optimization for industrial control applications using wireless sensor and actuator mesh networks," IEEE Transactions on Industrial Electronics, vol. 64, no. 4, pp. 3250-3259, April 2017.

[15] M. Neely, Stochastic Network Optimization with Application to Communication and Queueing Systems. Morgan and Claypool, 2010.

[16] Radiocommunication Sector of ITU, "P 1238-9: Propagation data and prediction methods for the planning of indoor radiocommunication systems and radio local area networks in the frequency range $300 \mathrm{MHz}$ to $100 \mathrm{GHz}$, Jun 2017, vol. ITU-R, Tech. Rep. 